\title{
Risco climático do feijão da seca no Estado de Goiás, sob preparo de solo convencional e plantio direto
}

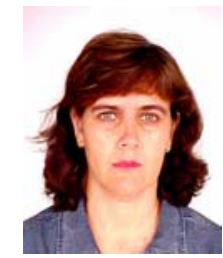

Elza J. L. Meireles ${ }^{1}$, Luís F. Stone ${ }^{2}$, Luciano de S. Xavier ${ }^{3}$ \& José A. A. Moreira ${ }^{4}$

1 Embrapa Café. Parque Estação Biológica - PqEB s/n, Edifício Sede da Embrapa - Plano Piloto, SI. 322.A, $3^{\circ}$ andar, CEP 70770-901, Brasília, DF. Fone: (61) 448-4566. E-mail: jacqueline.meireles@embrapa.br (Foto)

2 Embrapa Arroz e Feijão. Rod. Goiânia/Nova Veneza, km 12, CP 179, CEP 75375-000, Santo Antônio de Goiás, GO. Fone: (62) 533-2186. E-mail: stone@cnpaf.embrapa.br

${ }^{3}$ Bolsista da FINATEC. Fone: (62) 533-2125. E-mail: Ixavier@cnpaf.embrapa.br

${ }^{4}$ Embrapa Arroz e Feijão. Fone: (62) 533-2187. E-mail: jaloisio@cnpaf.embrapa.br

Protocolo 172 - 5/12/2001 - Aprovado em 6/2/2003

\begin{abstract}
Resumo: Na região dos Cerrados, os agricultores têm adotado o sistema plantio direto (SPD) como forma de proteger o ambiente e dar sustentabilidade à exploração agrícola. Neste sistema, a cobertura do solo pela palhada pode minimizar o risco climático das culturas de sequeiro, pela redução do déficit hídrico. O objetivo deste trabalho foi comparar o risco climático do feijão da "seca" no Estado de Goiás, sob preparo de solo convencional e plantio direto. Foram utilizados o modelo BIPZON para o cálculo do balanço hídrico e um sistema de informações geográficas para a espacialização dos resultados, além de identificadas as áreas de baixo, médio e alto risco climático para esta cultura, para o SPD, com 50 e $100 \%$ de cobertura do solo pela palhada e para o sistema convencional de preparo de solo. Verificou-se que a utilização do SPD causou aumento das áreas de menor risco e prolongamento do período favorável de semeadura a esta cultura, em relação ao sistema de preparo convencional. O efeito foi mais pronunciado com $100 \%$ de cobertura do solo.
\end{abstract}

Palavras-chave: Phaseolus vulgaris L., épocas de semeadura, déficit hídrico, palhada

\section{Climatic risk of rainfed beans in the State of Goiás, Brazil, under conventional and no-till systems}

\begin{abstract}
In the Cerrado Region, farmers have been adopting the no-till system, to protect the soil and give sustainability to agriculture. In the no-till system, soil covered by crop residues can minimize climatic risks by reducing water deficit of crops sown in the late season. The objective of this study was to compare the climatic risk of dry season common beans grown in the State of Goiás, under conventional and no-till systems. The BIPZON model was used to calculate the water balance. Geographic Information System was used for the characterization of the results in time and space. Areas of low, medium and high climatic risks were identified for the no-till system, with 50 and $100 \%$ of soil coverage by crop residues, as well as for the conventional till system. It was verified that the use of no-till system increased the areas with lower risks and extended the period of sowing for the bean crop in relation to conventional till system. The effect was higher with $100 \%$ soil covered with crop residues.
\end{abstract}

Key words: Phaseolus vulgaris L., sowing period, water deficit, crop residues

\section{INTRODUÇÃO}

Na região dos Cerrados, os agricultores têm adotado o sistema plantio direto (SPD) como forma de proteger o ambiente e dar sustentabilidade à exploração agrícola. Com base na ausência de movimentação do solo e na manutenção de resíduos orgânicos na sua superfície, o SPD provoca alterações na dinâmica da matéria orgânica e da atividade biológica modificando principalmente os processos intrínsecos do solo, provocando alterações na sua estrutura e na dinâmica físico-hídrica.
A palhada na superfície do solo altera a relação solo-água, pois previne a evaporação, reduzindo assim a taxa de evapotranspiração das culturas. Barros \& Hanks (1993) e Stone \& Moreira (2000) observaram maior eficiência do uso da água pelo feijoeiro no sistema de plantio direto com cobertura morta, em relação a outros sistemas de preparo do solo, sendo que os últimos autores verificaram economia de água variando de 14 a $30 \%$, dependendo do porte da planta de feijão. Andrade (2001), comparando o efeito de diversas percentagens de cobertura do solo pela palhada sobre o consumo de água do feijoeiro em 
plantio direto, verificou que o tratamento de $100 \%$ de cobertura propiciou economia de água de $27 \%$ em relação ao tratamento sem cobertura.

A cobertura do solo pode minimizar o risco climático das culturas de sequeiro, pela redução do déficit hídrico. Muitos trabalhos têm evidenciado que, em plantio direto, o conteúdo de água do solo é maior que em áreas cultivadas com preparo convencional (Sidiras et al., 1983; Salton \& Mielniczuk, 1995; Stone \& Silveira, 1999). Sidiras et al. (1983) verificaram que, em plantio direto, o solo reteve de 36 a 45\% mais água disponível para as culturas, reduzindo as perdas de água por evaporação e aumentando o armazenamento de água no solo. Desta maneira, com a determinação do coeficiente de cultura em função de diferentes porcentagens de cobertura do solo pela palhada, como realizado para o feijoeiro (Andrade, 2001), é possível realizar-se o zoneamento agroclimático desta cultura e, por comparação com o zoneamento feito para condição de preparo convencional do solo, medir o efeito da palhada na redução do déficit hídrico, mediante o aumento da percentagem de área com menor risco climático.

O objetivo deste trabalho foi comparar o risco climático do feijão da "seca", semeado nos meses de janeiro e fevereiro, no Estado de Goiás, sob preparo de solo convencional e plantio direto.

\section{MATERIAL E MÉTODOS}

Para cálculo do balanço hídrico, base decendial, utilizou-se o modelo BIPZON, desenvolvido por Franquin \& Forest (1977) já validado em vários trabalhos (Steinmetz et al., 1985; Assad, 1986; Meireles et al., 1995; Silva et al., 1994, 1997).

Neste modelo são utilizadas simplificações que, se contestadas do ponto de vista conceitual, podem ser contornadas, apresentando pequena margem de erro (inferior a 10\%) quando comparadas com as medições da umidade de solo obtidas pela sondagem de nêutrons e pelo método aerodinâmico do balanço de energia (Assad, 1987) - o que é aceitável tratando-se de simulação. Uma das premissas é de que o modelo considera o solo como compartimento isotrópico e monofásico, o que, na realidade, não ocorre. Outra premissa é a determinação dos coeficientes culturais que, por serem medidos em lisímetros e terem abrangência local, devem ser corrigidos sempre que se altere a referência espacial. Finalmente, a determinação da evapotranspiração real (ETr) é feita por uma equação polinomial de terceiro grau, desenvolvida por Eagleman (1971) dada na Eq. 1; trata-se de um modelo empírico, mas de fácil manuseio e aplicabilidade.

$$
\mathrm{ETr}=\mathrm{A}+\mathrm{BHR}-\mathrm{CHR}{ }^{2}+\mathrm{DHR}^{3}
$$

em que,

$$
\begin{array}{ll}
\text { A } & -0,732-0,05 \mathrm{ETm} \\
\text { B } & -4,97 \mathrm{ETm}-0,66 \mathrm{ETm}^{2} \\
\text { C } & -8,57 \mathrm{ETm}-1,56 \mathrm{ETm}^{2} \\
\text { D } & -4,35 \mathrm{ETm}-0,88 \mathrm{ETm}^{2} \\
\text { HR } & \text { - umidade do solo }
\end{array}
$$

Ressalta-se que o modelo utilizado considera a cultura do feijão da "seca" sem limitação nutricional e com o controle adequado de pragas, doenças e plantas daninhas.
As variáveis de entrada do modelo são:

\section{Precipitação pluvial diária}

Utilizaram-se séries com 15 anos de dados diários de chuva de 121 estações pluviométricas do Estado de Goiás, cujas informações foram fornecidas pela Agência Nacional de Energia Elétrica (ANEEL) e pelo Instituto Nacional de Meteorologia (INMET).

\section{Capacidade de armazenamento de água no solo}

Foram consideradas três capacidades de armazenamento de água no solo: baixa $(30 \mathrm{~mm})$, média $(40 \mathrm{~mm})$ e alta $(50 \mathrm{~mm})$.

\section{Coeficiente de cultura (Kc)}

Utilizaram-se os valores de Kc referentes ao sistema de preparo convencional (SPC) da cultura do feijoeiro, sem cobertura do solo, obtidos por Steinmetz (1984) e aqueles relacionados ao sistema de plantio direto (SPD) com 50 e $100 \%$ de cobertura do solo, obtidos por Andrade (2001) (Tabelas 1 e 2).

Tabela 1. Coeficiente de cultura $(\mathrm{Kc})$ para três fases do ciclo do feijoeiro, em Santo Antônio de Goiás, GO

\begin{tabular}{lcc}
\hline Fases da Cultura & Duração (dias) & Kc \\
\hline Germinação ao início da floração & 35 & 0,69 \\
Floração & 25 & 1,28 \\
Desenvolvimento de vagens à maturação & 20 & 1,04 \\
\hline Fonte: Steinmetz (1984) & &
\end{tabular}

Tabela 2. Coeficientes de cultura médios, em diferentes períodos do ciclo do feijoeiro, cultivar Pérola, em relação às porcentagens de cobertura morta, em Santo Antônio de Goiás, GO

\begin{tabular}{ccc}
\hline & \multicolumn{2}{c}{ Coeficiente de Cultura } \\
\cline { 2 - 3 } Dias Após a Emergência & \multicolumn{2}{c}{ Cobertura do Solo (\%) } \\
\cline { 2 - 3 } & 50 & 100 \\
\hline 20 & 0,54 & 0,36 \\
27 & 0,70 & 0,57 \\
34 & 0,85 & 0,91 \\
41 & 1,15 & 1,01 \\
48 & 1,13 & 0,98 \\
55 & 1,05 & 0,90 \\
62 & 0,97 & 0,60 \\
69 & 0,61 & 0,47 \\
76 & 0,47 & 0,37 \\
\hline
\end{tabular}

Fonte: Andrade (2001)

\section{Evapotranspiração potencial (ETp)}

A ETp foi estimada pela equação de Penman (1963) pelo INMET, para cerca de 30 estações climatológicas, distribuídas no Estado. Posteriormente, os valores de ETp foram espacializados e extrapolados para as regiões que não possuíam informações.

\section{Ciclo da cultivar de feijão da "seca" estudado}

Considerou-se uma cultivar de ciclo normal (90 d) em que o período crítico (floração-enchimento de grãos) é de $45 \mathrm{~d}$ (entre os $30^{\circ}$ e $75^{\circ} \mathrm{d}$ ).

Os balanços hídricos foram determinados no período compreendido entre janeiro e fevereiro, considerando-se os primeiro, segundo e terceiro decêndios de cada mês.

Uma das saídas mais importantes do modelo é a relação $\mathrm{ETr} / \mathrm{ETm}$, ou seja, evapotranspiração real e evapotranspiração 
máxima, que expressa a quantidade de água que a planta irá consumir e o total necessário para garantir a sua máxima produtividade.

Para cada localidade calcularam-se os valores médios de ETr/ETm da fase de florescimento/enchimento de grãos, para cada ano. Uma vez determinados esses valores, efetuou-se uma análise freqüencial para $80 \%$ de ocorrência pois, sendo uma freqüência mais conservadora, indica se a relação ETr/ ETm é maior ou igual a determinado valor para 8 em cada 10 anos, ou seja, em $80 \%$ dos anos. Este estudo não é probabilístico, retrata o que ocorreu no passado e, na faixa freqüencial de $80 \%$, espera-se, no máximo, $20 \%$ de situações atípicas, não explicitadas na análise. Isto quer dizer que é possível se encontrar situações em que a ETr/ETm seja muito inferior ao calculado, com chances de $20 \%$ de ocorrência.

Para a caracterização do risco climático ao cultivo do feijão da "seca", no Estado de Goiás, foram estabelecidas três classes de ETr/ETm, conforme Silva et al. (1999):

- ETr/ETm $\geq 0,60$ - a cultura do feijão da "seca" está exposta a um baixo risco climático.

- 0,6>ETr/ETm $\geq 0,50$ - a cultura do feijão da "seca" está exposta a um risco climático médio.

- ETr/ETm<0,50 - a cultura do feijão da "seca" está exposta a um alto risco climático.

Os valores calculados para definir o risco climático foram espacializados utilizando-se um sistema de informações geográficas.

Para a espacialização dos resultados, foram adotados os seguintes procedimentos: digitação de arquivo de pontos (em formato ASCII) organizados em três colunas, com latitude, longitude e valores de relação ETr/ETm, com $80 \%$ de freqüência de ocorrência; transformação das coordenadas geográficas em coordenadas de projeção cartográfica utilizadas (no caso, projeção policônica); leitura do arquivo de pontos; organização das amostras; e geração de uma grade regular (grade retangular, regularmente espaçada de pontos, em que o valor da cota de cada ponto é estimado a partir da interpolação de um número de vizinhos mais próximo).

Por se tratar de uma análise bidimensional, na qual as variações de ETr/ETm foram espacializadas em função do tempo, desconsiderando-se os efeitos orográficos, o interpolador escolhido foi aquele que mais se aproximou de um resultado linear. Assim, foi selecionado o interpolador 1, que considerou a média dos $\mathrm{N}$ pontos mais próxima por quadrante da grade regular, com peso $\mathrm{W}=1 / \mathrm{d}^{\mathrm{n}}$, em que a distância euclidiana do ponto amostrado ao ponto interpolado (d) foi igual a 4 e o expoente da função distância (n) igual a 3. Essas foram as opções que apresentaram o menor erro médio, dentre as combinações possíveis.

Convertidos os dados e feitas as transformações necessárias, a imagem foi fatiada e reclassificada. Verificados os erros e ajustados os valores das interpolações, foram confeccionadas as figuras definindo-se as regiões, conforme o risco climático; posteriormente, calcularam-se as áreas de baixo, médio e alto risco climático para o feijão da "seca" no Estado de Goiás, para o SPD, com 50 e $100 \%$ de cobertura do solo pela palhada, e para o sistema convencional de preparo do solo.

\section{RESULTADOS E DISCUSSÃO}

Observa-se, na Tabela 3, o risco climático para o feijão da "seca", semeadura nos meses de janeiro e fevereiro, no Estado
Tabela 3. Áreas percentuais sob diferentes riscos climáticos para o feijão da "seca”, para o Estado de Goiás, considerandose solos com diferentes capacidades de armazenamento de água (A) 30, (B) 40 e (C) $50 \mathrm{~mm}$ e os sistemas de preparo de solo convencional e plantio direto

\begin{tabular}{ccc|}
\multirow{2}{*}{ Semeadura } & \multicolumn{2}{c|}{$\begin{array}{c}\text { Áreas (\%) Conforme o Risco } \\
\text { Climático do Feijão da "Seca" }\end{array}$} \\
\cline { 2 - 3 } Risco & Kc - SPC & Kc - SPD ${ }^{2}$ \\
\cline { 3 - 4 } & & $50 \%$ Cob. $\quad 100 \%$ Cob. \\
\hline
\end{tabular}

A. $30 \mathrm{~mm}$

\begin{tabular}{|c|c|c|c|c|}
\hline & Alto & 34,81 & 0,42 & 0 \\
\hline \multirow[t]{2}{*}{01 a $10 / 01$} & Médio & 50,07 & 2,75 & 0,73 \\
\hline & Baixo & 15,12 & 96,83 & 99,27 \\
\hline \multirow{4}{*}{11 a $20 / 01$} & Alto & 66,39 & 0,34 & 0,01 \\
\hline & Médio & 31,46 & 5,67 & 0,91 \\
\hline & Baixo & 2,15 & 93,99 & 99,08 \\
\hline & Alto & 94,33 & 2,24 & 0,31 \\
\hline \multirow[t]{3}{*}{21 a $30 / 01$} & Médio & 5,67 & 8,56 & 0,99 \\
\hline & Baixo & 0 & 89,20 & 98,70 \\
\hline & Alto & 99,88 & 8,47 & 1,08 \\
\hline \multirow[t]{3}{*}{01 a $10 / 02$} & Médio & 0,12 & 38,76 & 6,18 \\
\hline & Baixo & 0 & 52,77 & 92,74 \\
\hline & Alto & 100,00 & 91,70 & 54,61 \\
\hline \multirow[t]{3}{*}{11 a $20 / 02$} & Médio & 0 & 6,90 & 27,82 \\
\hline & Baixo & 0 & 1,40 & 17,57 \\
\hline & Alto & 100,00 & 99,96 & 99,81 \\
\hline \multirow[t]{2}{*}{21 a $28 / 02$} & Médio & 0 & 0,04 & 0,17 \\
\hline & Baixo & 0 & 0 & 0,02 \\
\hline \multicolumn{5}{|l|}{ B. $40 \mathrm{~mm}$} \\
\hline \multirow{4}{*}{01 a $10 / 01$} & Alto & 15,87 & 0,14 & 0,03 \\
\hline & Médio & 32,12 & 0,89 & 0,20 \\
\hline & Baixo & 52,01 & 98,97 & 99,77 \\
\hline & Alto & 37,52 & 0,12 & 0 \\
\hline \multirow[t]{3}{*}{11 a $20 / 01$} & Médio & 47,47 & 2,18 & 0,11 \\
\hline & Baixo & 15,01 & 97,70 & 99,89 \\
\hline & Alto & 78,86 & 0,84 & 0,24 \\
\hline \multirow[t]{3}{*}{21 a $30 / 01$} & Médio & 20,12 & 4,81 & 0,56 \\
\hline & Baixo & 1,02 & 94,35 & 99,20 \\
\hline & Alto & 99,47 & 3,77 & 0,40 \\
\hline \multirow[t]{3}{*}{01 a $10 / 02$} & Médio & 0,42 & 15,21 & 1,22 \\
\hline & Baixo & 0,11 & 81,02 & 98,38 \\
\hline & Alto & 100,00 & 67,29 & 15,26 \\
\hline \multirow[t]{3}{*}{11 a $20 / 02$} & Médio & 0 & 24,28 & 28,44 \\
\hline & Baixo & 0 & 8,43 & 56,30 \\
\hline & Alto & 100,00 & 99,89 & 99,41 \\
\hline \multirow[t]{2}{*}{21 a $28 / 02$} & Médio & 0 & 0,11 & 0,47 \\
\hline & Baixo & 0 & 0 & 0,12 \\
\hline \multicolumn{5}{|l|}{ C. $50 \mathrm{~mm}$} \\
\hline \multirow{4}{*}{01 a $10 / 01$} & Alto & 9,24 & 0,07 & 0 \\
\hline & Médio & 14,93 & 0,38 & 0 \\
\hline & Baixo & 75,83 & 99,55 & 100,00 \\
\hline & Alto & 17,55 & 0,04 & 0 \\
\hline \multirow[t]{3}{*}{11 a $20 / 01$} & Médio & 33,11 & 0,41 & 0,16 \\
\hline & Baixo & 49,34 & 99,55 & 99,84 \\
\hline & Alto & 43,30 & 0,52 & 0,11 \\
\hline \multirow[t]{3}{*}{21 a $30 / 01$} & Médio & 43,31 & 2,53 & 0,27 \\
\hline & Baixo & 13,39 & 96,95 & 99,62 \\
\hline & Alto & 98,49 & 1,02 & 0,09 \\
\hline \multirow[t]{3}{*}{01 a $10 / 02$} & Médio & 1,23 & 3,83 & 0,34 \\
\hline & Baixo & 0,28 & 95,15 & 99,55 \\
\hline & Alto & 100,00 & 30,92 & 4,51 \\
\hline \multirow[t]{3}{*}{11 a $20 / 02$} & Médio & 0 & 42,91 & 13,58 \\
\hline & Baixo & 0 & 26,17 & 81,91 \\
\hline & Alto & 100,00 & 99,87 & 97,34 \\
\hline 21 a $28 / 02$ & Médio & 0 & 0,13 & 2,21 \\
\hline & Baixo & 0 & 0 & 0,45 \\
\hline
\end{tabular}

SPC - Sistema de preparo convencional; ${ }^{2}$ SPD - Sistema plantio direto 
de Goiás, para solos com diferentes capacidades de armazenamento de água (30, 40 e $50 \mathrm{~mm})$ considerando-se os sistemas de preparo de solo convencional (SPC) e o plantio direto (SPD) com 50 e $100 \%$ de cobertura morta.

Pela Tabela 3A, verifica-se que para as condições de SPC em um solo com baixa capacidade de armazenamento de água (30 $\mathrm{mm})$ as áreas de alto risco são muito superiores àquelas de baixo risco, principalmente à medida que se atrasa a semeadura. Como exemplo, para a semeadura do feijoeiro efetuada entre 11 e 20 de janeiro, verifica-se a ocorrência de áreas de baixo risco em apenas 2,15\%; médio risco em $31,46 \%$ e alto risco em $66,39 \%$ do Estado. A partir de $1^{\circ}$ de fevereiro, as áreas de alto risco foram superiores a $99 \%$; entretanto, aumentando-se o armazenamento de água, solos de média e alta capacidade de armazenamento, 40 e $50 \mathrm{~mm}$, respectivamente, constata-se que a ocorrência de áreas de alto risco climático foi reduzida gradativamente para as semeaduras realizadas entre $1^{\circ}$ e 20 de janeiro, prevalecendo maiores áreas de baixo e médio risco em todo o Estado de Goiás (Tabelas 3B e C).

Caso o feijão da "seca" fosse cultivado em SPD com apenas $50 \%$ de cobertura morta, pela Tabela 3 poder-se-ia observar que, para as semeaduras efetuadas entre 01/01 a 10/02, a ocorrência de áreas com alto risco climático se reduziria consideravelmente, prevalecendo áreas de baixo risco em quase todo o Estado correspondendo, em média, a 83,2, 93,0 e 97,8\%, respectivamente, para a capacidade de armazenamento de água no solo, de baixa, média e alta.

O aumento da cobertura do solo para $100 \%$, num solo de baixa capacidade de armazenamento de água, favoreceu o aumento das áreas de baixo risco climático no Estado, sendo, em média, $97,44 \%$ do total, e também ocorreu o prolongamento do período de semeadura de $1^{\circ}$ de janeiro a 10 de fevereiro. A partir de 11/02, a condição de alto risco climático foi predominante no Estado dificultando, portanto, o cultivo do feijão da "seca", em virtude da redução da disponibilidade hídrica para a cultura na fase de floração-enchimento de grãos, considerada a mais crítica em demanda de água. Para os solos de média e alta capacidade de armazenamento de água, o alto risco se torna evidente na maior parte do Estado, no período entre 21 e 28/02; portanto, as datas de semeadura para o feijão da "seca" com menores riscos climáticos para os solos com $100 \%$ de cobertura morta e maior capacidade de armazenamento de água (40 e 50 mm) se concentraram entre 01/01 e 20/02.

A Figura 1 exemplifica a distribuição das áreas de baixo, médio e alto risco climático do feijão da "seca" nesse Estado, para a semeadura entre 11 e 20 de janeiro, considerando-se o solo de média capacidade de armazenamento de água (40 mm) e sistemas de preparo convencional do solo e plantio direto (50 e 100\% de cobertura morta).

Verifica-se, no SPD, aumento da ocorrência de áreas de menor risco climático e prolongamento das datas de semeadura mais propícias ao cultivo do feijão da "seca" em relação ao SPC. Portanto, a adoção do SPD para o cultivo do feijão da "seca" com utilização de cobertura morta no solo, possibilitará maior proteção da camada superficial do solo, o que, aliado à maior estabilidade estrutural, permitirá maior infiltração de água no solo em relação ao sistema de preparo convencional, ocasionando menor perda de água por escoamento superficial.
A.

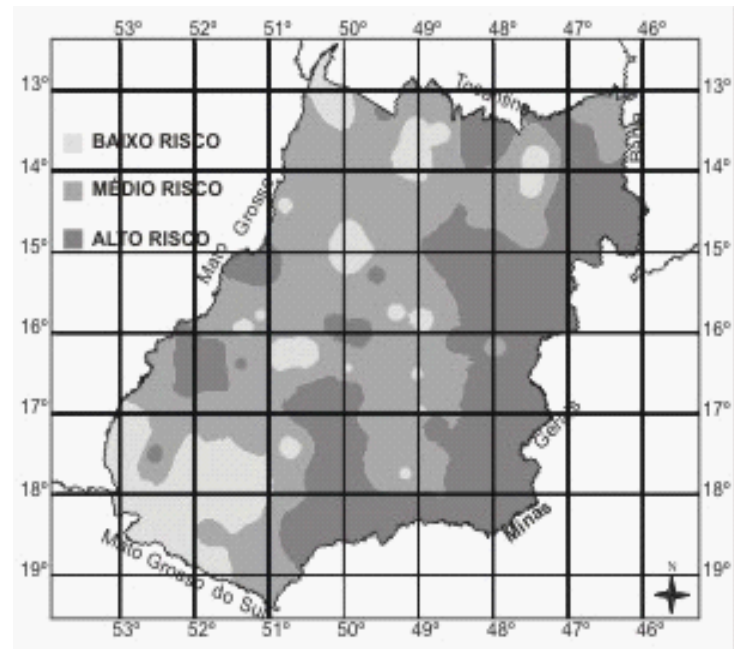

B.

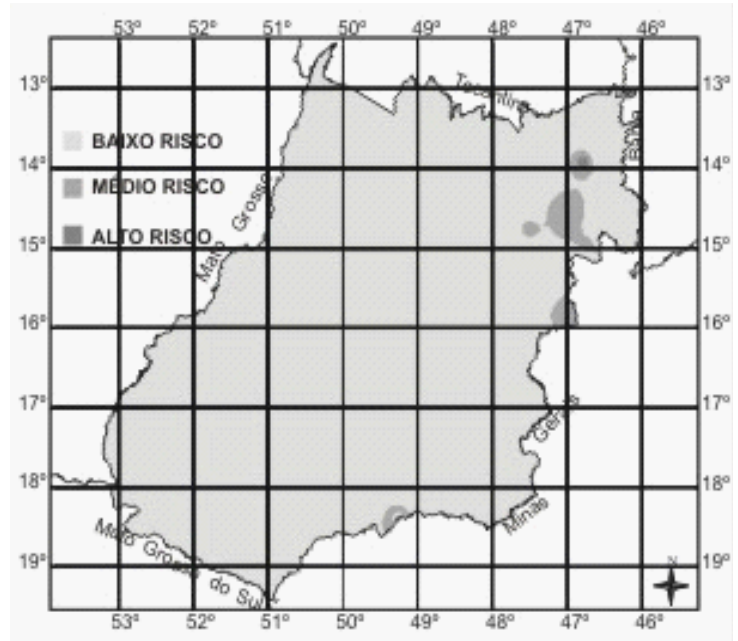

C.

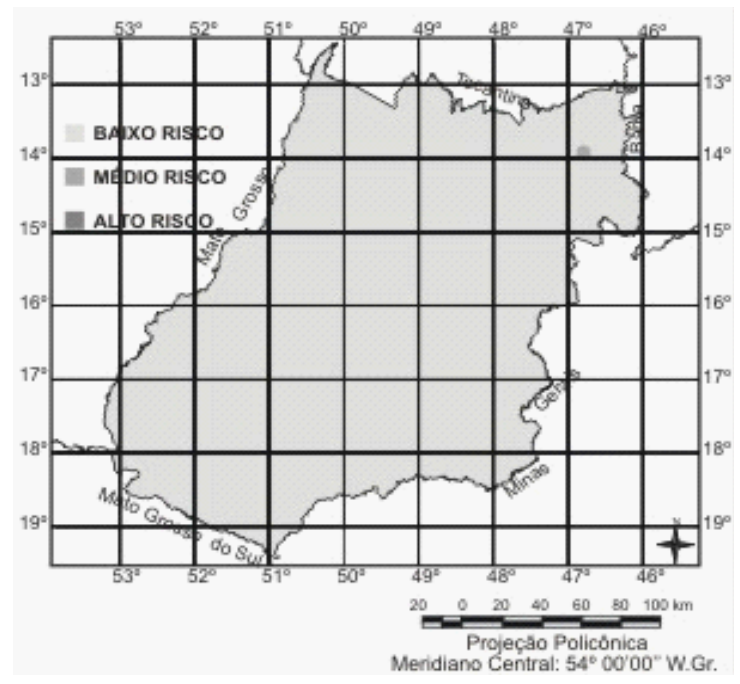

Figura 1. Áreas de baixo, médio e alto risco climático para o feijão da "seca", para semeadura entre 11 e 20/01, e solo de média capacidade de armazenamento de água (40 mm) em (A) sistema de preparo de solo convencional, (B) sistema plantio direto (50\% de cobertura morta) e (C) sistema plantio direto (100\% de cobertura morta), no Estado de Goiás 
Além disso, o SPD, por sua vez, proporcionará aumento da capacidade de armazenamento de água no perfil, por ser um sistema que provoca menor revolvimento do solo (Vieira, 1981; Stone \& Silveira, 1999). A cobertura do solo pela palhada também causa redução da taxa de evaporação do solo mantendo-se, assim, maior disponibilidade de água no perfil. Andrade (2001) verificou que a evapotranspiração do feijoeiro apresentou valores menores, à medida que aumentou a porcentagem de cobertura do solo pela palhada. Barros \& Hanks (1993) observaram que a cobertura morta aumentou a eficiência do uso da água do feijoeiro, em todos os níveis de irrigação estudados.

Com base nesses resultados, sugere-se que nos próximos estudos de risco climático relacionados ao cultivo do feijão da "seca" seja mencionado o sistema de preparo de solo utilizado, se SPC ou SPD contribuindo, deste modo, para uma política agrícola e uma alocação de recursos mais racionais.

\section{CONCLUSÕES}

1. Na análise de risco climático do feijão da "seca" no Estado de Goiás, a utilização do sistema plantio direto proporcionou aumento da ocorrência de áreas com menor risco e prolongamento do período favorável de semeadura, em relação ao sistema preparo convencional.

2. Esses efeitos foram mais evidentes quanto maior a porcentagem da cobertura do solo pela palhada.

\section{LITERATURA CITADA}

Andrade, R. da S. Consumo relativo de água pelo feijoeiro (Phaseolus vulgaris L.) no Sistema Plantio Direto em função da percentagem de cobertura morta do solo. Lavras: UFLA, 2001. 52p. Dissertação Mestrado

Assad, E.D. Simulation de irrigation et du drainage pour les pluviales de riz de maiz en soils de bas-fonds a Brasília. Montpellier: IRAT, 1986. 10p. IRAT. Memories et Travaux, 13

Assad, E.D. Utilisation des satellites meteorologiques pour le suivi agroclimatique des cultures en zone sahelienne: Cas du Senegal. Montpellier: Université des Sciences et Techniques du Languedoc, 1987. 258p. Tese Doutorado

Barros, L.C.G.; Hanks, R.J. Evapotranspiration and yield of beans as affected by mulch and irrigation. Agronomy Journal, Madison, v.85, p.692-697, 1993.

Eagleman, A.M. An experimentaly derived model for actual evapotranspiration. Agricultural Meteorology, Amsterdam, v.8, n.4/5, p.385-409, 1971.

Franquin, P.; Forest, F. Des programmes pour l'évaluation et l'analyse frequentielle des termes du bilan hydrique. L'Agronomie Tropicale, Paris, v.32, n.1, p.7-11, 1977.
Meireles, E.J.L.; Silva, S.C. da; Assad, E.D.; Lobato, E.J.V.; Bezerra, H.S.; Evangelista, B. A.; Moreira, L.; Cunha, M.A.C. da. Zoneamento agroclimático para o arroz de terras altas no Estado de Tocantins. Goiânia: EMBRAPA-CNPAF, 1995. 72p. Documentos, 58

Penman, H.L. Vegetation and hydrology. Harpenden: Commonwealth Bureau of Soils, 1963. 125p. Technical Communication, 53

Salton, J.C.; Mielniczuk, J. Relações entre sistemas de preparo, temperatura e umidade de um Podzólico Vermelho-Escuro de Eldorado do Sul (RS). Revista Brasileira de Ciência do Solo, Campinas, v.19, n.2, p.313-319, 1995.

Sidiras, N.; Derpsch, R.; Mondardo, A. Influência de dife- rentes sistemas de preparo do solo na variação da umidade e rendimento da soja, em Latossolo Roxo distrófico (Oxi-sol). Revista Brasileira de Ciência do Solo, Campinas, v.7, p.103106, 1983.

Silva, S.C. da; Assad, E.D.; Lobato, E.J.V.; Sano, E.E.; Steinmetz, S.; Bezerra, H.S.; Cunha, M.A.C. da; Silva, F.A.M. da. Zoneamento agroclimático para o arroz de sequeiro no Estado de Goiás. Brasília: EMBRAPA-SPI, 1994. 80p. Documentos, 43

Silva, S.C. da; Meireles, E.J.L.; Assad, E.D.; Xavier, L.S.; Cunha, M.A.C. da. Caracterização do risco climático para a cultura do arroz de terras altas no Estado de Mato Grosso. Goiânia: EMBRAPA-CNPAF, 1997. 18p. EMBRAPA-CNPAF. Documentos, 76

Silva, S.C. da; Meireles, E.J.L.; Xavier, L.S.; Alves, S.F.; Barsi, R.O. Zoneamento agroclimático para o cultivo do feijão da "seca" em Goiás. Santo Antônio de Goiás: EMBRAPACNPAF, 1999. 52p. Documentos, 94

Steinmetz, S. Evapotranspiração máxima no cultivo do feijão de inverno. Goiânia: EMBRAPA-CNPAF, 1984. 4p. Pesquisa em Andamento, 47

Steinmetz, S.; Reyniers, F.N.; Forest, F. Evaluation of the climatic risk on upland rice in Brazil. In: Colloque "Resistance A La Sécheresse En Millieu Intertropicale: Quelles Recherches Our Le Moyen Terme?", 1984, Dakar. Proceedings... Paris: CIRAD, 1985. p.43-54.

Stone, L.F.; Moreira, J.A.A. Efeitos de sistemas de preparo do solo no uso da água e na produtividade do feijoeiro. Pesquisa Agropecuária Brasileira, Brasília, v.35, n.4, p.835-841, 2000.

Stone, L.F.; Silveira, P.M. da. Efeitos do sistema de preparo na compactação do solo, disponibilidade hídrica e comportamento do feijoeiro. Pesquisa Agropecuária Brasileira, Brasília, v.34, n.1, p.83-91, 1999.

Vieira, M.J. Propriedades físicas do solo. In: IAPAR (Londrina, PR). Plantio direto no Estado do Paraná. Londrina: IAPAR, 1981.p.19-32. Circular, 23 